\title{
Efficiency of Thyroid Function Test in the Prediction of Atrial Fibrillation Following Open Heart Surgery
}

\author{
Selen Öztürk ${ }^{1}$, Ilyas Kayacioglu ${ }^{1}$, Yavuz Sensoz ${ }^{1}$, Kemal Özdemir $^{1}$ and Ibrahim Öztürk ${ }^{2}$ \\ ${ }^{1}$ Department of Cardiovascular Surgery, Siyami Ersek Chest and Cardiovascular Surgery Education and Research Hospital, Istanbul, \\ Turkey \\ ${ }^{2}$ Department of Anesthesiology, Göztepe Education and Research Hospital, Istanbul, Turkey
}

\begin{abstract}
Objective: To analyse the efficiency of thyroid function tests (thyroid stimulating hormone, thyroxine and tri-iodothyronine) on prediction of postoperative atrial fibrillation.

Study Design: Meta-analysis.

Place of Study: Siyami Ersek Chest and Cardiovascular Surgery Education and Research Hospital, Istanbul, Turkey.

Methodology: Literature review was carried out in PubMed, Science Direct and Ovid Database. No date limitations was applied. Trials, which evaluated the preoperative levels of thyroid stimulating hormone, thyroxine and tri-iodothyronine in cardiac surgery patients, were included. Only the articles in English language were reviewed. We evaluated the results with fix or random effect models according to the presence of heterogeneity (I2 >25\%).

Results: Five hundred and forty-seven articles were obtained after screening of databases. After checking over the titles and abstracts, five trials were included which covered 380 patients and complied with inclusion criteria. According to results of analysis, there was no significant relationship between postoperative atrial fibrillation and thyroid stimulating hormone (SMD: 0.38, 95\% Cl -0.46-1.23, $\mathrm{p}=0.375$ ), and also thyroxine (SMD: 0.006, 95\% Cl -0.29-0.30, p=0.966). However, tri-iodothyronine (SMD: $-1.06,95 \% \mathrm{Cl}-2.08--0.03, \mathrm{p}=0.04)$ was correlated with development of atrial fibrillation. Heterogeneity was observed in three parameters (I ${ }^{2}$; for TSH: $92.45 \%$, for T3: $93.08 \%$ and for T4: $31.78 \%$ ).

Conclusion: Preoperative levels of tri-iodothyronine was an effective parameter for predicting postoperative atrial fibrillation after cardiac surgery, but there was a need of larger trials for eliminating heterogeneity for all of the parameters of thyroid function tests.
\end{abstract}

Key Words: Cardiac surgery, Atrial fibrillation, Thyroid stimulating hormone, Thyroxine, Tri-iodothyronine.

How to cite this article: Öztürk S, Kayacioglu I, Sensoz Y, Özdemir K, Öztürk I. Efficiency of Thyroid Function Test in the Prediction of Atrial Fibrillation Following Open Heart Surgery. J Coll Physicians Surg Pak 2020; 30(07):740-744.

\section{INTRODUCTION}

Both hypothyroidism and hyperthyroidism may affect cardiac function and health. Hypothyroidism have many hemodynamic effects on cardiovascular system, such as, decreasing cardiac output and arterial compliance, increasing systemic vascular resistance, risk of coronary artery disease, risk of atherosclerosis and diastolic pressure, and also narrowing pulse pressure. ${ }^{1}$ Hypothyroidism can decrease and thyroid hormones can increase heart rate due to various mechanisms, such as: endothelial dysfunction and decrease of nitric oxide production, vascular smooth muscle relaxation and cardiac contractility. ${ }^{2}$

Correspondence to: İbrahim Öztürk, Department of Anesthesiology, Göztepe Education and Research Hospital, Istanbul, Turkey

E-mail: drozturk28@gmail.com

Received: January 28, 2020; Revised: July 13, 2020;

Accepted: July 17, 2020

DOI: https://doi.org/10.29271/jcpsp.2020.07.740
On the other hand, hyperthyroidism affects cardiovascular hemodynamics. Heart failure and dilated cardiomyopathy may occur because of hyperthyroidism. ${ }^{3}$ Effective treatment of hyperthyroidism can prevent heart failure. Moreover, prevention of atrial fibrillation (AF) and thromboembolic events are very important during hyperthyroidism treatment. ${ }^{3}$

Atrial fibrillation is the most common arrhythmia that can cause heart failure and also stroke. ${ }^{4}$ Selmer et al. compared the euthyroid individuals with overt and subclinical hypothyroid/hyperthyorid patients in a large population cohort study. ${ }^{5}$ They found the incidence risk ratios for AF were 0.67 for overt hypothyroidism, 0.87 for subclinical hypothyroidism, 1.31 for subclinical hyperthyroidism, and 1.42 for overt hyperthyroidism. The authors concluded that the risk of AF was closely associated with thyroid activity: low risk with overt hypothyroidism and high risk with hyperthyroidism. They also found the AF risk was thyroid stimulating hormone (TSH) level dependent.

The aim of this study was to determine whether preoperative levels parameters (free thyroxine-fT4, free triiodothyronine-fT3 and TSH) of thyroid function tests can predict postoperative new onset $\mathrm{AF}$ (POAF) after cardiac surgery or not. 
Table I: Summary of studies.

\begin{tabular}{|c|c|c|c|c|c|c|c|c|}
\hline & \multirow{2}{*}{ Year } & \multirow{2}{*}{$\begin{array}{c}\text { POAF } \\
\mathbf{n}\end{array}$} & \multirow{2}{*}{$\begin{array}{c}\text { Total } \\
\mathbf{n}\end{array}$} & \multirow{2}{*}{ Design } & \multirow[b]{2}{*}{ Type of surgery } & \multicolumn{3}{|c|}{ Weights of studies in analysis (\%) } \\
\hline & & & & & & TSH & FreeT3 & FreeT4 \\
\hline Kokkonen et al. & 2005 & 19 & 44 & Prospective & CABG + valve surgery & 19.79 & 25.27 & 18.50 \\
\hline Dietrich et al. & 2015 & 17 & 39 & Prospective & CABG + valve surgery & 18.33 & 21.75 & 17.18 \\
\hline Cerillo et al. & 2003 & 33 & 107 & Prospective & Isolated CBABG & 20.87 & 26.41 & 31.40 \\
\hline Gode et al. & 2016 & 15 & 90 & Prospective & Isolated CBABG & 20.06 & - & - \\
\hline Özsin et al. & 2018 & 50 & 100 & Prospective* & Isolated CBABG & 20.96 & 26.58 & 32.93 \\
\hline
\end{tabular}

Table II: Results of analysis.

\begin{tabular}{|c|c|c|c|c|c|c|c|c|c|c|c|}
\hline \multirow{3}{*}{$\begin{array}{l}\text { Variables } \\
\text { Thyroid stimulating hormone }\end{array}$} & \multicolumn{4}{|c|}{ Results } & \multicolumn{4}{|c|}{ Heterogeneity } & \multirow{3}{*}{$\begin{array}{c}\text { Publication bias } \\
\text { Tau }^{2} \\
0.84 \\
\end{array}$} & \multicolumn{2}{|c|}{ Fail safe analysis } \\
\hline & SMD & & & $\mathbf{p}$ & $\mathbf{Q}$ & df & $\mathbf{p}$ & $\begin{array}{ll}I^{2} & (\%)\end{array}$ & & 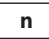 & $\mathbf{p}$ \\
\hline & 0.38 & -0.46 & 1.23 & 0.375 & 31.30 & 4 & $<0.001$ & 92.45 & & 4 & 0.014 \\
\hline Tri-iodothyronine & -1.06 & -2.08 & -0.03 & $0.04 *$ & 43.36 & 3 & $<0.001$ & 93.08 & 0.99 & 45 & $<0.001$ \\
\hline Thyroxine & 0.006 & -0.29 & 0.30 & 0.966 & 4.28 & 3 & 0.23 & 31.78 & $0.029 *$ & 0 & 0.44 \\
\hline
\end{tabular}

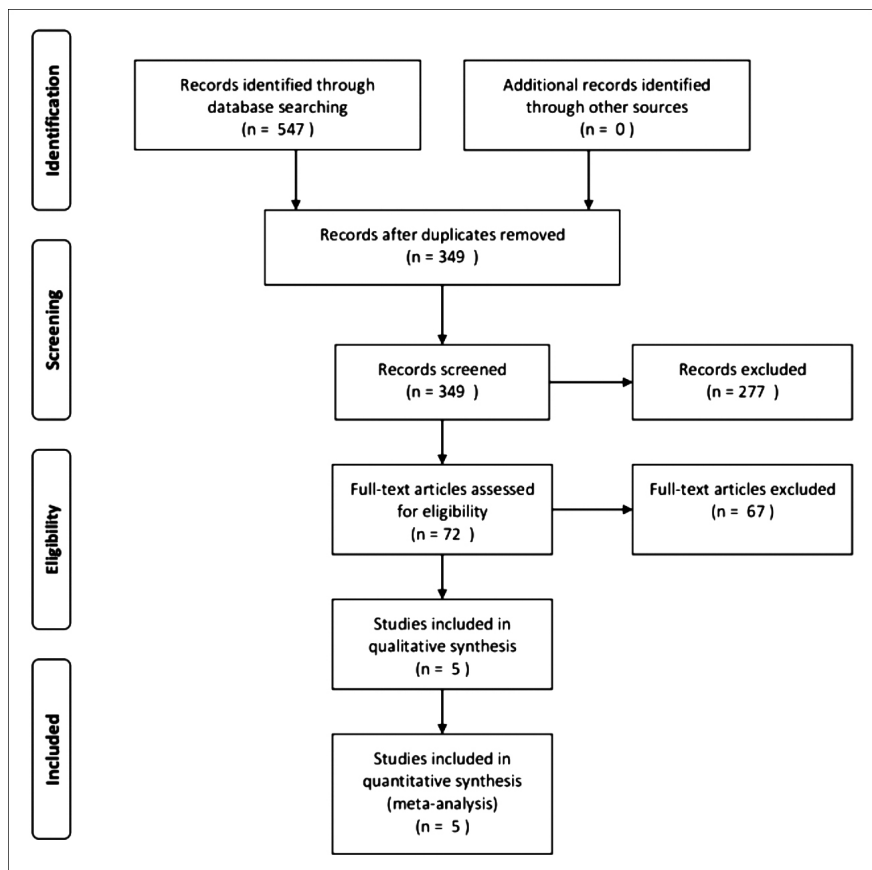

Figure 1: Flow diaphragm of database searching.

\section{METHODOLOGY}

Databases were searched according to the guidelines reported by Moher et al. to investigate whether preoperative thyroid function tests can predict the occurrence of atrial fibrillation after heart surgery or not. ${ }^{6}$ The database was searched until 20th December, 2019. There was no limitation for publication date. PubMed, Science Direct and Ovid was used as electronic database. Manual search was not employed. Keywords or combinations of them (heart surgery, coronary artery bypass, cardiac surgery, atrial fibrillation, thyroid function test, thyroid stimulating hormone, thyroxine and tri-iodothyronine) were used. Language was limited to English. The other languages were excluded. The study designs and patient numbers were not limiting factors.

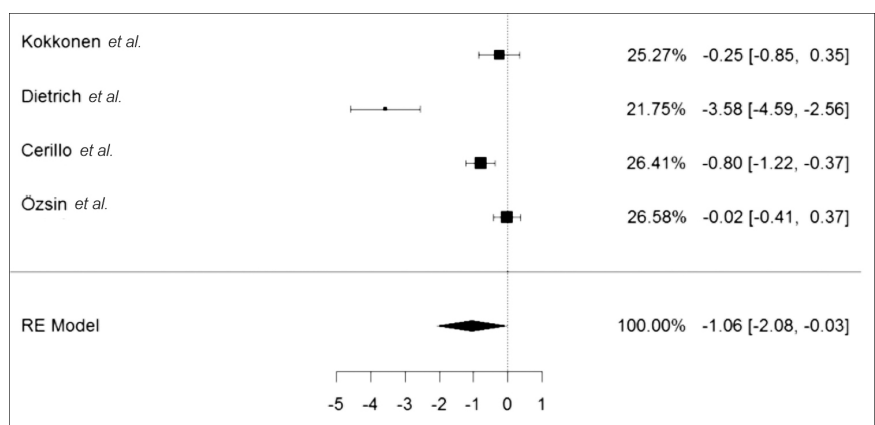

Figure 2: Forest plot for free T3.

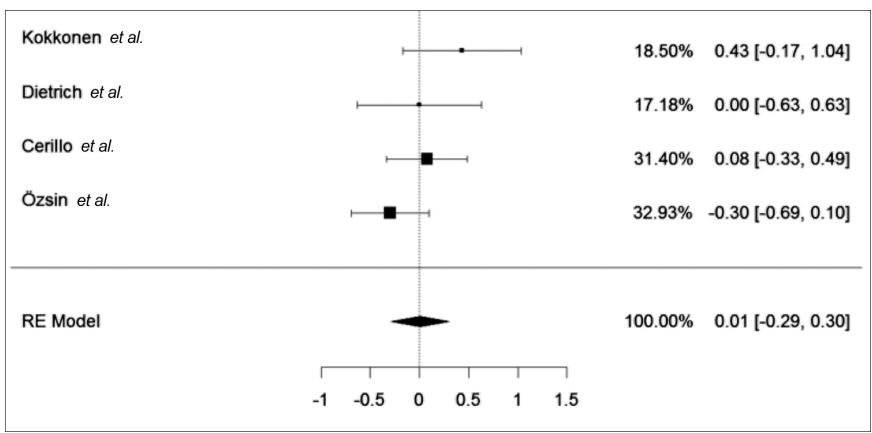

Figure 3: Forest plot for free T4.

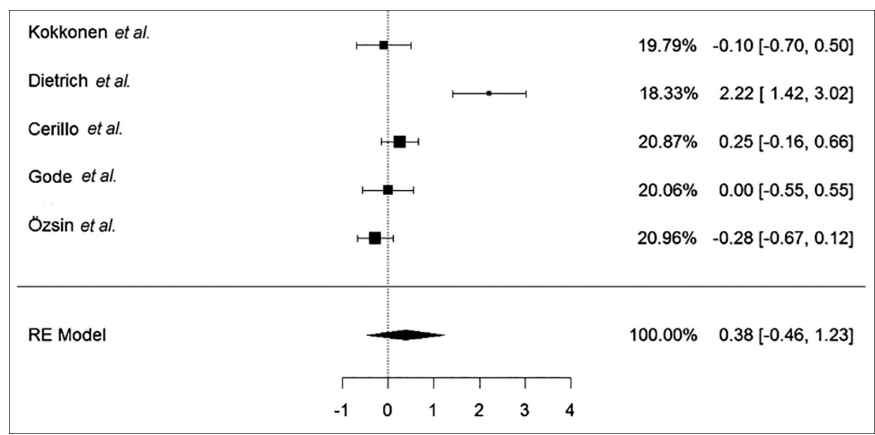

Figure 4: Forest plot for TSH.

Inclusion criteria were (i) clinical trials, (ii) open heart surgery (iii) article in English, (iv) coronary artery or valve 
surgery. Exclusion criteria were (i) case reports and case series (ii) non-English articles, (iii) experimental studies, and (iv) peripheral vascular surgery. The related articles not containing the knowledge about preoperative levels of thyroid stimulating hormone, thyroxine and triiodothyronine, were also excluded. Articles reporting data with figures were excluded.

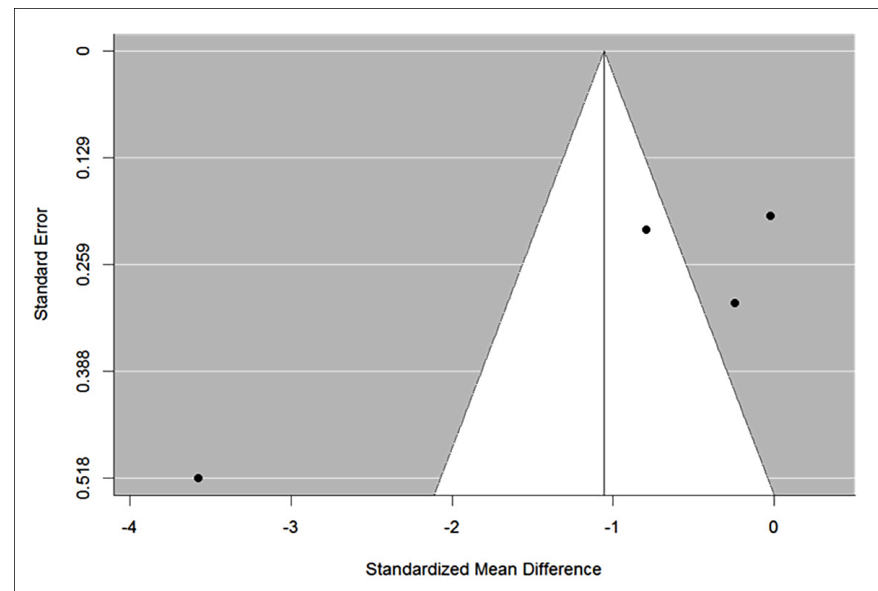

Figure 5: Funnel plot for free T3.

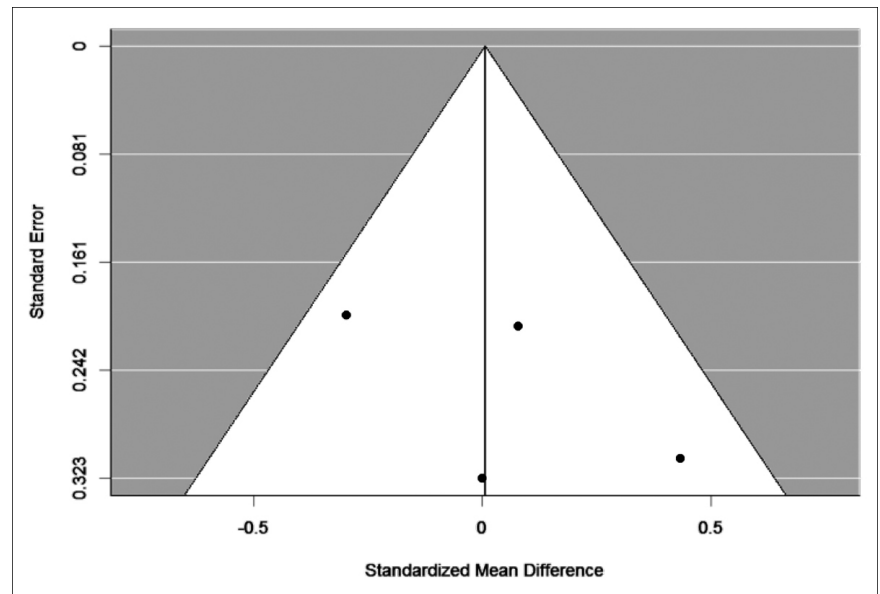

Figure 6: Funnel plot for free T4.

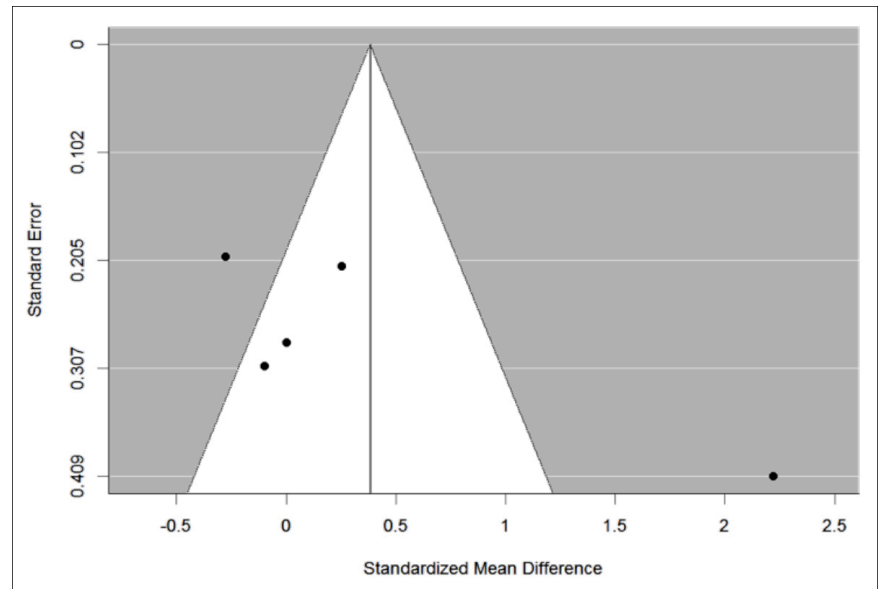

Figure 7: Funnel plot for TSH.
Three reviewers (S.Ö., Y.Ş. and I.Ö.) extracted data from related trials. Publication information (first author's name, publication year, sample size, number of patients with POAF and also with no-POAF, in each group) were extracted. Disagreement was resolved by the other authors (I.K. and K.Ö.). The mean, standard deviation and sample sizes were recorded as data.

Jamovi ${ }^{\circledR}$ software was used for statistical analysis. The standard mean difference (SMD) and 95\% confidence interval $(\mathrm{Cl})$ was used for analysis. $l^{2}$ statistics was used for evaluation of heterogeneity. Presence of heterogeneity was agreed as significant if $I^{2} \geq 25 \%$. We evaluated the possible reasons of heterogeneity with the analysis of moderators. Meta-analysis was applied by using fixed or random effect models. Random effect model was performed for the presence of heterogeneity $\left(I^{2}>25 \%\right)$ and fixed effect model for the absence of heterogeneity $\left(I^{2}<25 \%\right)$. Begg test and funnel plot was used for publication bias.

\section{RESULTS}

Three hundred and fifty-eight articles were identified through database search. Three hundred and forty-nine records remained after removing the duplicated articles. Authors excluded 349 records after checking the titles and abstracts. The records were screened for relation; and 277 trials were excluded. Seventy-two full text articles were assessed for eligibility to the main question of our investigation. Sixty-seven trials without the detailed data were excluded. Five articles, summarised in Table I, were included to quantitative synthesis. ${ }^{7-11}$ Flow diaphragm of database search startegy is shown in Figure 1; and demographical data of trials, weights of studies due to results of analysis for three parameters are summarised in Table I.

At the end of the analysis, only fT3 could predict POAF after cardiac surgery $(p=0.04)$. Random effect model was used for evalaution because of the heterogeneity of studies $\left(\mathrm{I}^{2}\right.$; for TSH: $92.45 \%$, for fT3: $93.08 \%$ and for fT $4: 31.78 \%)$. Percentage of heterogeneity was smallest for fT4. Summary of analysis is shown in Table II; and forest plots of analysis were shown in Figures 2-4. We could determine the reason of heterogeneity as type of surgery, only for fT4. $\mathrm{I}^{2}$ test was $\% 0$ in combined valve and CABG surgery.

For fT4, the publication bias was significant (tau ${ }^{2}=0.029$ ). For $\mathrm{ft} 3$ and $\mathrm{TSH}, \operatorname{tau}^{2}$ was not significant (>0.05). The funnel plots were shown in Figures 5-7.

The possible numbers of studies that we might missed out during database search (fail-safe $N$ ) were 45 for TSH, 4 for fT3 and 0 for fT4, according to Rosenthal approach. 


\section{DISCUSSION}

It was found that preoperative levels of fT3 can predict POAF after cardiac surgery. Because of the limited numbers of articles in this issue, the rate of heterogeneity were very high for thyroid function test parameters. All the trials had a prospective design. However, only Ozsin et al. study was randomised controlled trial. ${ }^{10}$ We could not determine the main reason of heterogeneity, but we thought reason might be nonrandomisation of samples. The limited number of randomised controlled study (only one) prevented the analsis of heterogeneity during statistical analysis.

AF may ocur after different types of surgeries, but the incidence of POAF is greatest in cardiac surgery. POAF is recorded up to $40 \%$ in coronary artery bypass grafting surgery, $63 \%$ in valve surgery and $11 \%$ in heart transplantation. ${ }^{12,13}$ On the other hand, many risk factors are defined for POAf after cardiac surgery. Age is the most reported and widely accepted risk factor ${ }^{14}$ The possible mechanism of AF due to the aging process is leading to a loss of myocardial fibers, increasing fibrosis and collagen deposition in the atria. ${ }^{15}$ Except the age, one can classify the risk factors for POAF in two groups: cardiovascular and noncardiovascular factors. ${ }^{16}$ Cardiovascular factors such as congestive heart failure, coronary artery disease, noncoronary vascular disease, prior history of AF/other arrhythmias, left atrial enlargement, hypertension, and left ventricular dysfunction; and non-cardiovascular factors such as chronic obstructive pulmonary disease, hyperthyroidism, chronic kidney disease, male gender, diabetes mellitus, Caucasian race, high cholesterol, and obesity can cause POAF.

Between the two opposite status of thyroidal function, hyperthyroidism is more agreed predisposing medical condition for POAF than hypothyroidism. Frost et al. diagnosed atrial fibrillation (8.3\%) within 30 days from the diagnosis of hyperthyroidism, among 40,628 patients. ${ }^{17}$ On the other hand, contribution of hypothyroidism for POAF is contraversial. In the earlier studies, possible correlation between AF and hypothyroidism has been suggested, but not proven. ${ }^{18}$ Barnes et al. found that $50 \%$ of patients received amiodarone and abnormal thyroid function tests due to amiodarone. ${ }^{18}$ Because of that they concluded routine screening for hypothyroidism has not seemed justifiable from their data.

Krahn et al. demonstrated that abnormal TSH level was common in new onset AF patients, but clinical thyroid disease was uncommon. ${ }^{19}$ Therefore, they suggested routine TSH screening of patients at higher risk of having undiagnosed clinical thyroid disease.

In two recent retrospective studies, hypothyroidism was associated with development of POAF after cardiac surgery. ${ }^{20,21}$ Those studies were performed in 821 and 626 patients, respectively. Cerillo et al. have shown low fT3 concentration has been agreed as an univariate predictor of POAF development. ${ }^{9}$ Kokkonen et al. have performed an investigation in patients 65 years or older, and they had taken blood samples three times at preoperative, fourth postoperative hour and third postoperative month. ${ }^{7}$ Only the fourth postoperative hour fT3 values were different between POAF and non-POAF groups. No differences were observed for TSH or fT4. Authors demonstrated that fT3 at the fourth hour, age and number of grafts were independent predictors of POAF.

In an experimental trial, Zhang et al. concluded that both hypothyroidism and hyperthyroidism have led to increased AF vulnerability in a rat thyroidectomy model. ${ }^{22}$ According to this trial, normal thyroid hormone levels are required to to prevent cardiac arrhythmias and $\mathrm{AF}$.

The main limitation of this meta-analysis was the language restriction of articles. The second limitation was small sample sizes of studies, and inclusion of only a single trial.

\section{CONCLUSION}

According to the result of this analysis, preoperative lower levels of fT3 is an important predictor of POAF. However, that result depends on the smaller sample size trials. Therefore, there is a need for larger studies for a common outcome such as POAF.

\section{CONFLICT OF INTEREST:}

The authors declare no conflicts of interest with respect to the authorship and/or publication of this article.

\section{AUTHORS' CONTRIBUTION:}

SÖ, IK, YS, KÖ, IÖ: Concept, design, data collection, analysis, literature search, writing manuscript and critical review.

\section{REFERENCES}

1. Klein I, Danzi S. Thyroid disease and the heart. Circulation 2007; 116(15):1725-35. doi: 10.1161.

2. Osuna PM, Udovcic M, Sharma MD. Hyperthyroidism and the Heart. Methodist Debakey Cardiovasc J 2017; 13(2):60-3. doi: 10.14797/mdcj-13-2-60.

3. Udovcic M, Pena RH, Patham B, Tabatabai L, Kansara A. Hypothyroidism and the Heart. Methodist Debakey Cardiovasc J 2017; 13(2):55-9. doi: 10.14797/mdcj-13-2-55.

4. Selmer C, Olesen JB, Hansen ML, Lindhardsen J, Olsen AM, Madsen JC, et al. The spectrum of thyroid disease and risk of new onset atrial fibrillation: A large population cohort study. BMJ 2012; 345:e7895. doi: 10.1136/bmj.e7895.

5. Wilson C. Thyroid function: Thyroid dysfunction and risk of atrial fibrillation. Nat Rev Endocrinol 2013; 9(2):63. doi: 10.1038/nrendo.2012.239. 
6. Moher D, Shamseer L, Clarke M, Ghersi D, Liberati A, Petticrew $M$, et al. Preferred reporting items for systematic review and meta-analysis protocols (PRISMA-P) 2015 statement. Syst Rev 2015; 4(1):1. doi: 10.1186/ 2046-4053-4-1.

7. Kokkonen L, Majahalme S, Kööbi T, Virtanen V, Salmi J, Huhtala $\mathrm{H}$, et al. Atrial fibrillation in elderly patients after cardiac surgery: Postoperative hemodynamics and low postoperative serum triiodothyronine. J Cardiothorac Vasc Anesth 2005; 19(2):182-7. doi: 10.1053/j.jvca.2005.01.028.

8. Dietrich JW, Müller P, Schiedat F, Schlömicher M, Strauch J, Chatzitomaris $A$, et al. Nonthyroidal Illness syndrome in cardiac Illness involves elevated concentrations of 3,5diiodothyronine and correlates with atrial remodeling. Eur Thyroid J 2015; 4(2):129-37.

9. Cerillo AG, Bevilacqua S, Storti S, Mariani M, Kallushi E, Ripoli $A$, et al. Free triiodothyronine: A novel predictor of postoperative atrial fibrillation. Eur J Cardiothorac Surg 2003; 24(4):487-92. doi: 10.1016/s1010-7940(03)00396-8.

10. Özsin KK, Sanrı US, Toktaş F, Kahraman N, Yavuz Ş. Effect of plasma level of vitamin $d$ on postoperative atrial fibrillation in patients undergoing isolated coronary artery bypass grafting. Braz J Cardiovasc Surg 2018; 33(3):217-223. doi: 10.21470/1678-9741-2017-0214.

11. Gode S, Aksu T, Demirel A, Sunbul M, Gul M, Bakır I, et al. Effect of vitamin $D$ deficiency on the development of postoperative atrial fibrillation in coronary artery bypass patients. J Cardiovasc Thorac Res 2016; 8(4):140-6. doi: 10.15171/ jcvtr.2016.29.

12. Pires LA, Wagshal AB, Lancey R, Huang SK. Arrhythmias and conduction disturbances after coronary artery bypass graft surgery: Epidemiology, management, and prognosis. Am Heart J 1995; 129(4):799-808. doi: 10.1016/0002-8703 (95)90332-1.

13. Creswell LL, Schuessler RB, Rosenbloom M, Cox JL. Hazards of postoperative atrial arrhythmias. Ann Thorac Surg 1993; 56(3):539-49. doi: 10.1016/0003-4975(93)90894-n.

14. January CT, Wann LS, Alpert JS, Calkins H, Cigarroa JE, Cleveland JC Jr, et al. American college of cardiology american heart association task force on practice guidelines. 2014 aha/acc/hrs guideline for the management of patients with atrial fibrillation: A report of the american college of cardiology/american heart association task force on practice guidelines and the heart rhythm society. J Am Coll Cardiol 2014; 64(21):e1-76. doi: 10.1161/CIR. 0000000000000665.

15. Philip I, Berroeta C, Leblanc I. Perioperative challenges of atrial fibrillation. Curr Opin Anaesthesiol 2014; 27(3): 344-52. doi: 10.1097/ACO.0000000000000070.

16. Greenberg JW, Lancaster TS, Schuessler RB, Melby SJ. Postoperative atrial fibrillation following cardiac surgery: A persistent complication. Eur J Cardiothorac Surg 2017; 52(4):665-72. doi: 10.1093/ejcts/ezx039.

17. Frost L, Vestergaard P, Mosekilde L. Hyperthyroidism and risk of atrial fibrillation or flutter: A population-based study. Arch Intern Med 2004; 164(15):1675-8. doi: 10.1001/ archinte.164.15.1675.

18. Barnes DJ, O'Connor JD, Bending JJ. Hypothyroidism in the elderly: Clinical assessment versus routine screening. $\mathrm{Br}$ J Clin Pract 1993; 47(3):123-7.

19. Krahn AD, Klein GJ, Kerr CR, Boone J, Sheldon R, Green M, et al. How useful is thyroid function testing in patients with recent-onset atrial fibrillation? The canadian registry of atrial fibrillation Investigators. Arch Intern Med 1996; 156 (19):2221.

20. Worku B, Tortolani AJ, Gulkarov I, Isom OW, Klein I. Preoperative hypothyroidism is a risk factor for postoperative atrial fibrillation in cardiac surgical patients. J Card Surg 2015; 30(4):307-12. doi: 10.1111/jocs.12513.

21. Jaimes MC, Torrado LAA, Reyes NFS, Mackenzie JC, Mallarino JPU. Hypothyroidism is a risk factor for atrial fibrillation after coronary artery bypass graft. Braz J Cardiovasc Surg 2017; 32(6):475-80. doi.org/10.21470/1678-9741-2017-0080.

22. Zhang Y, Dedkov El, Teplitsky D, Weltman NY, Pol CJ, Rajagopalan $\mathrm{V}$, et al. Both hypothyroidism and hyperthyroidism increase atrial fibrillation inducibility in rats. Circ Arrhythm Electrophysiol 2013; 6(5):952-9. doi: 10.1161/ CIRCEP.113.000502. 